being refereed within 45 days with an average of around a fortnight. The most rapid acceptance was the paper on the Caret trial, which was approved on the day it was submitted, or even perhaps the paper by Prasad and Arjun, which had no receipt or acceptance date so presumably was accepted in no time at all.

Scientists continually bemoan the plethora (if that is indeed the right word) of new journals, but it seems inevitable that more and more will be published. If so, then one hopes they will be as well presented as Anti-Cancer Drugs, which should develop into a respectable research journal.

T. A. Connors is in the Medical Research Council Toxicology Unit, MRC Research Council Laboratories, Woodmansterne Road, Carshalton, Surrey SM5 4EF, UK.

\section{Good exposure}

\section{J. Kinlen}

Cancer Causes and Control: An International Journal of Studies of Cancer in Human Populations. Editor B. MacMahon. Rapid Communications of Oxford. 6/yr. £222, \$399.

CANCER kills an appreciable proportion of the world's population (in North America and Europe, around 1 in 5) and many more suffer from its curable forms. The study of its causes in human populations (epidemiology) is therefore of considerable importance. Cancer Causes and Control was launched in July 1990 under the editorship of the distinguished epidemiologist Brian MacMahon.

As the opening editorial reminds us, epidemiology has already made great strides in indicating the roles played in human cancer by tobacco, asbestos, infection with hepatitis B virus, sunlight, ionizing radiation and certain chemicals. Indeed, if exposure to these were eliminated (or reduced in the case of ultraviolet light), cancer rates would probably be reduced by a third or even more.

Traditionally, papers in the field of cancer epidemiology have appeared in general medical, cancer or epidemiological journals. There is no doubt that the specialized nature of many papers makes them unsuited to a general readership, whereas cancer journals are mainly devoted to laboratory or clinical studies. Cancer Causes and Control has the distinction of being the first journal to be devoted exclusively to cancer epidemiology. Emphasis is to be placed on speed of publication - an aim so far achieved, all papers having been published within two months of acceptance. The coverage of subjects is wide, but contributions in the first two volumes have concerned one of the most important current problems in cancer causation - whether dietary fat causes cancer of the breast and other organs. I cannot think of another journal that would allow so extensive an airing of differing views. This and the speed of publication augur well for its future, although it is expensive.

$L: J$. Kinlen is Director of the Cancer Research Campaign Cancer Epidemiology Research Group, Department of Public Health, University of Oxford, The Radcliffe Infirmary, Oxford OX2 6HE, UK.

\section{Bright future}

D. P. Lane

Seminars in Cancer Biology. Guest editors. Saunders. 6/yr. US $\$ 130$, e/sewhere $\$ 155$ (institutional); US $\$ 130$, elsewhere $\$ 102$ (industrial); US $\$ 63$, elsewhere $\$ 84$ (personal).

WHY do people agree to write reviews? I suppose the full range of human emotions are involved. At the more base level they are flattered to be asked, the deadline seems about two lifetimes away and it gives them a good opportunity to rewrite history, emphasizing the unique importance of their own contribution. It might look good on their curriculum vitae and do wonders for their ranking in the Science Citation Index. They might even get paid as well. Nobler motives might include a desire to try and pull the field together and make it more comprehensible to others. At best the author can bring fresh insight to an area that will colour future research.

Why do people read reviews? The need comes from the vast size of the primary literature, from its bias, and from a need to avoid arcane acronyms. Move slightly out of your own field and the primary literature can become impenetrable. Certainly it becomes very difficult to make a critical judgement about rival claims. A striking example of this is the recent attempts of one and all to understand the amusing serology of idiotypes in the Weaver et al. paper at the centre of the Baltimore affair. Good reviews can bridge the gap; they need to be independent, up-to-date and critical. At the technical level they need to be short and provide an entry into the relevant primary literature.

Seminars in Cancer Biology is a much better series than I expected. There are several established rivals in this field but the series has found a niche because of its format. Each issue uses a new guest editor, an expert in the field, and covers a specific topic. The reviews are short and show signs of good editing. Thank- fully references are given in full. The coordination between volumes is generally good, though Fos and Jun, for example, are reviewed three times in the six volumes I looked at, exceeding even my great admiration for these proteins.

So if the idea is valid and the organization good, what about the reviews themselves? I looked at two volumes in my field, that on transcription factors edited by Nick Jones and that on nuclear oncogenes edited by Gerard Evan. Both of these were really very good. The reviews were up-to-date and pitched, I thought, at just the right level. Generally they were written by well-known people in the field and with some authority. I would have liked to see a bit more direct talking when discrepancies in the field were discussed, but overall the standard was high. The other volumes, more peripheral to my own work, were also encouraging. Those on antibodies, on the epidermal-growth-factor receptor family and on proteases, were at the right level and were well coordinated.

There is a need for this kind of series, as it forms a bridge between the exhaustive traditional review and the kind of summary that marks out the Current Biology series. As more of molecular oncology drifts towards the real world of treatment, this series will prove helpful to keep us all up-to-date. If the present standard and momentum can be maintained then it has a bright future.

D. P. Lane is in the Department of Biochemistry, Medical Sciences Institute, University of Dundee, Dundee DD1 $4 H N$, UK.

\section{In company}

\section{Michael T. Lotze}

Biotechnology Therapeutics. Editors Steven Gillis and Arthur Ammann. Dekker. 4/yr. $\$ 225$ (institutional); $\$ 117.50$ (personal).

ONE of the secrets to the rapid development of interleukin-2 biology was the availability of a rapid, extremely sensitive bioassay using the CTLL line identified by Steven Gillis. This approach to identifying biological activities, developing assays and then cloning the genes encoding them has recently been duplicated with many other growth factors and cytokines and represents one of Gillis's lasting legacies. I remember running into him at a conference almost a dozen years ago after he had made the rounds of traditional academia at Dartmouth, Memorial Sloan-Kettering and the Fred Hutchinson Cancer Center, when he indicated that he was inclined to start a company to develop biotechnology further. He, together with Larry 